\title{
A Double-Integral Type of Indirect Sliding Mode Controllers for Power Converters
}

\author{
Siew-Chong Tan, Y. M. Lai, Chi K. Tse, and Chi-Kin Wu
}

\begin{abstract}
The steady-state regulation error in power converters that use the conventional hysteresis-modulationbased sliding mode controller can be suppressed through the incorporation of an additional integral term of the state variables into the controller. However, it is found that with the indirect type of sliding mode controller (derived based on the equivalent control approach), the same approach of integral sliding mode control is ineffective in alleviating the converter's steady-state error. Moreover, the error increases as the converter's switching frequency decreases. This paper presents an in-depth study of the phenomenon and offers a solution to the problem. Specifically, it is proposed that an additional double-integral term of the controlled variables to be adopted for constructing the sliding surface of indirect sliding mode controllers. Experimental results are provided for verification.
\end{abstract}

Index Terms-Power converters, sliding mode control, nonlinear controller, pulsewidth modulation, double-integral sliding mode.

\section{INTRODUCTION}

The practical use of sliding mode (SM) controllers in power converters is often challenged by two major concerns: the non-constant operating frequency of the SM controller, and the presence of steady-state error in the regulation. For the first concern, various possible methods of fixating the switching frequency of SM controllers have been proposed. These include the use of adaptive strategies [1], the incorporation of constant timing functions or circuitries [2], [3], and the indirect implementation of the SM controllers [4]-[6]. As for the second concern, it has been widely known that the steady-state errors of sliding mode controlled systems can be effectively suppressed through the use of an additional integral term of the state variables in the SM controller [3], [4], [7]-[9]. This method is known as integral sliding mode control. When incorporated, the consequence is an SM controlled system 1) with motion equation of the same order as the original system; and 2) with better robustness and regulation property than the traditional SM controlled system [10].

Interestingly, in a recent attempt to implement a type of fixed-frequency indirect SM controllers, which is based on pulsewidth-modulation (PWM), for the DC-DC converters [5], [6], [11], it is noted that the adoption of the integral sliding mode (ISM) control scheme in these controllers can reduce, but not fully alleviate the steady-state error of the converters. Our investigation shows that this is due to the imperfect steady-state error correction method of the PWMbased ISM controllers. The problem is common to all types of indirect ISM controllers derived from the equivalent control method [4], [5], [6], [11]-[13]. Moreover, the steady-state error increases as the converter's switching frequency decreases and that it can be significantly large at a low switching frequency. Since increasing the order of the controller of a system generally improves the steady-state accuracy [14], we explore the possibility of alleviating the steady-state regulation error of the indirect ISM controlled converters by increasing the order of the indirect ISM controllers using an additional integral term. Hence, the objective of this paper is to present an in-depth study of the phenomenon, and to report the effectiveness of the proposed solution - the use of doubleintegral state variables for constructing the sliding surface of indirect SM controllers for power converters, in suppressing the steady-state error. Various aspects of the proposed solution including its design and implementation are discussed in term of the fixed-frequency PWM-based (indirect) SM controller. Experimental results are provided to validate the solution.

\section{PRoblem Identification}

\section{A. Review of Hysteresis-Modulation-Based Sliding Mode Con- trollers}

Conventional direct SM controllers based on hysteresismodulation (HM) are implemented through the real-time computation of the state variables to generate a suitable profile of the state variable's trajectory, which is then enforced to track a desired sliding surface to the equilibrium state [10]. Since SM control achieves order reduction, it is typically sufficient to have an SM controller of $n-1^{\text {th }}$ order for achieving stable control of an $n^{\text {th }}$ order converter. For instance, a common form of the SM controller for an $n^{\text {th }}$ order converter adopts a switching function

$$
u= \begin{cases}u^{+} & \text {when } S>\kappa \\ u^{-} & \text {when } S<-\kappa\end{cases}
$$

where $\kappa$ is a parameter controlling the switching frequency of the system, and $S$ is the instantaneous state variable's trajectory of reduced order, which is expressed as

$$
S=\sum_{i=1}^{n-1} \alpha_{i} x_{i},
$$

where $\alpha_{i}$ for $i=1$ to $n-1$ denotes the sets of the control parameters, i.e., sliding coefficients. Under the configuration $\kappa=0$, it is typically assumed in the SM control theory that the system operates ideally at an infinite switching frequency with no steady-state error. However, this is not true in practice. 
Practical non-ideality limits the switching frequency to be a finite quantity. Yet, if finite-frequency or fixed-frequency SM controllers are to be employed, the robustness and regulation properties of the converter system under the order-reduced SM controller will be deteriorated. Steady-state errors are therefore present.

A good method of suppressing these errors is to introduce an additional integral term of the state variables to the SM controller, which transforms it into an ISM controller (also known as full-order SM controller) [10]. Such an ISM controller can be obtained by modifying (2) into

$$
S=\sum_{i=1}^{n-1} \alpha_{i} x_{i}+\alpha_{n} \int \sum_{i=1}^{n-1} x_{i} d t
$$

where the additional state variable (as compared to (2)) is basically the integral term of all other existing state variables. Notably, it is the component $\int x_{i} d t$ which directly nullifies the steady-state errors of the respective state variables, i.e., $x_{i}$. However, when it comes to the indirect implementation of the ISM controller, the effectiveness of the integral control in alleviating the steady-state error deteriorates. The following section discusses why the ISM controller succeeds in alleviating the steady-state error in the direct (HM) form, but fails in the indirect (PWM) form.

\section{B. Review of Indirect Sliding Mode Controllers}

For implementation of SM controller in the indirect form, the original control law must be translated [5], [6]. This is based on an approach known as the equivalent control method, which assumes the invariance conditions that during SM operation, $S=0$ and $\dot{S}=0$. From such an assumption, an equivalent control signal $u_{\text {eq }}$ can be derived in terms of the respective state variables. Hence, the state variable's trajectory $S$ is indirectly formulated to track the desired sliding surface through the construction of the control signal $u_{\text {eq }}$. This makes it an indirect approach of ensuring SM operation as compared to the HM approach, which directly formulates $S$ to track the sliding surface.

To derive the equivalent control, the time differentiation of (3) is first derived, i.e.,

$$
\dot{S}=\sum_{i=1}^{n-1} \alpha_{i} \dot{x}_{i}+\alpha_{n} \sum_{i=1}^{n-1} x_{i}
$$

Equating $\dot{S}=0$ and solving for $u_{\mathrm{eq}}$ gives the general form

$$
u_{\mathrm{eq}}=G\left(\dot{x}_{1}, \dot{x}_{2}, \ldots, \dot{x}_{n-1}, x_{1}, x_{2}, \ldots, x_{n-1}\right)
$$

where $0<u_{\mathrm{eq}}<1$ is a function of the state variables $\dot{x}_{i}$ and $x_{i}$ for $i=1,2, \ldots, n-1$. In the implementation of PWM-based (indirect) SM controller, the control signal $u_{\text {eq }}$ is constructed through a pulsewidth modulator using a constant frequency ramp signal $v_{\text {ramp }}$ and a feedback control signal $v_{\mathrm{c}}$, where $u_{\text {eq }}=\frac{v_{\mathrm{c}}}{\hat{v}_{\text {ramp }}}$. Hence, both $v_{\text {ramp }}$ and $v_{\mathrm{c}}$ are functions of the state variables $\dot{x}_{i}$ and $x_{i}$. It is important to pinpoint that the indirect construction of $S$ using the indirect approach (such as PWM) uses state variables of one time derivative order lower than the original HM-based ISM controller (see equation (3)).
This explains why the steady-state error correction succeeds in HM-based ISM controller but fails in the indirect ISM controller, and why the problem is particularly severe when the switching frequency is low.

\section{Analytical Explanation for the Presence of Steady-State Error in Indirect ISM Controlled Converter}

In the case of the direct (HM-based) ISM controller, the sliding surface constructed comprises the integral elements of the steady-state errors, i.e, $\int x_{i} d t$. Recall that $\int x_{i} d t$ is a component that directly accumulates the existing steadystate errors. Hence, when the state variable's trajectory $S$ is directed to track the sliding surface to a point of equilibrium, the steady-state errors are automatically alleviated. With this process of closed-loop steady-state-error-correction feedback, the switching frequency will have little influence on the magnitude of the steady-state errors present in the HM-based ISM controlled converter.

However, for the indirect ISM controller, the variables $\int x_{i} d t$ are not explicitly reflected in the control signal (see equation (5)). Instead, these integral functions are embedded in the sliding surface, of which the required error corrections are indirectly computed using the state variables $\dot{x}_{i}$ and $x_{i}$. Since there is no direct integral signal $\int x_{i} d t$ that corrects the errors of the state variables, the capability of the correction is then dependent on the accuracy of the indirect integral computation. However, such computations are openloop processes which contain finite steady-state errors that cannot be eliminated. Hence, with steady-state errors present in the computation, steady-state errors will be present in the controlled variables. Naturally, this problem will be further aggravated if the switching frequency reduces.

\section{PRoposed Solution}

It is well known that the increased order of the controller improves the steady-state accuracy of the system, but aggravates the stability problem [14]. An additional double-integral term of the state variables, i.e., $\int\left[\int x_{i} d t\right] d t$ for $i=1,2, \ldots, n-1$, is therefore introduced to correct the error of the indirect integral computation in the indirect ISM controllers. By adding an integral closed-loop to alleviate the steady-state error of the indirect integral computation, the steady-state errors of the controlled state variables are indirectly alleviated. This is the so-called double-integral (indirect) sliding mode (DISM) controller proposed in this paper. Notably, the solution is simple and straightforward. But its use in practical design is never reported and requires investigation and validation.

In its general direct HM form, the proposed DISM controller takes the switching function (1) where

$S=\sum_{i=1}^{n-1} \alpha_{i} x_{i}+\alpha_{n} \int \sum_{i=1}^{n-1} x_{i} d t+\alpha_{n+1} \iint \sum_{i=1}^{n-1} x_{i} d t d t$

Clearly, this controller is of one order higher than the original converter system. Its time differentiation

$$
\dot{S}=\sum_{i=1}^{n-1} \alpha_{i} \dot{x}_{i}+\alpha_{n} \sum_{i=1}^{n-1} x_{i}+\alpha_{n+1} \int \sum_{i=1}^{n-1} x_{i} d t
$$


is likewise an order higher than the ISM (full-order) controller (4). By solving $\dot{S}=0$, it is not difficult to see that the equivalent control $u_{\text {eq }}$ is a function $G$ of the state variables $\dot{x}_{i}, x_{i}$, and $\int x_{i} d t$. Here, the additional term $\int x_{i} d t$ (as compared to ISM controller) is resulted from the double-integral term $\int\left[\int x_{i} d t\right] d t$ introduced by the DISM controller. It is interesting to see that by directly correcting the steady-state errors $x_{i}$, the original objective of introducing this component to correct the error of the indirect integral computation so that the steady-state errors of the controlled variables are alleviated is inherently met. The proposed DISM configuration easily resolves the problem of steady-state errors in indirect ISM controlled converters.

\section{Application of Double-InTEgral SLIDING Surface TO PWM-BASEd INDIRECT SLIDING Mode CONTROLlER}

This section discusses the application of the proposed solution, i.e., DISM configuration, to the PWM-based SM controller for the current controlled boost converter [11].

\section{A. Controller Formulation}

The proposed DISM controller for boost converters uses the switching function $u=\frac{1}{2}(1+\operatorname{sign}(S))$ and the sliding surface

$$
S=\alpha_{1} x_{1}+\alpha_{2} x_{2}+\alpha_{3} x_{3}+\alpha_{4} x_{4}
$$

where $u$ represents the logic state of power switch $S_{\mathrm{W}}$, and $\alpha_{1}, \alpha_{2}, \alpha_{3}$, and $\alpha_{4}$ represent the desired sliding coefficients. Here, the controlled state variables are the current error $x_{1}$, the voltage error $x_{2}$, the integral of the current and the voltage errors $x_{3}$, and the double integral of the current and the voltage errors $x_{4}$, which are expressed as

$$
\left\{\begin{array}{l}
x_{1}=i_{\text {ref }}-i_{L} \\
x_{2}=V_{\text {ref }}-\beta v_{\mathrm{o}} \\
x_{3}=\int\left[x_{1}+x_{2}\right] d t \\
x_{4}=\int\left(\int\left[x_{1}+x_{2}\right] d t\right) d t
\end{array},\right.
$$

where $i_{\text {ref }}=K\left[V_{\text {ref }}-\beta v_{\mathrm{o}}\right]$ and $K$ is the amplified gain of the voltage error. Additionally, the following converter parameters are assumed: $C, L$, and $r_{\mathrm{L}}$ denote the capacitance, inductance, and instantaneous load resistance respectively; $V_{\text {ref }}, v_{\mathrm{i}}$, and $v_{\mathrm{o}}$ denote the reference, instantaneous input, and instantaneous output voltages respectively; $\beta$ denotes the feedback network ratio; $i_{\text {ref }}, i_{L}, i_{C}$, and $i_{r}$ denote the instantaneous reference, inductor, capacitor, and output currents, respectively.

Substituting the boost converter's behavioral models under CCM into the time differentiation of (9) gives the dynamical model of the proposed system as

$$
\left\{\begin{array}{l}
\dot{x_{1}}=\frac{d\left[i_{\mathrm{ref}}-i_{L}\right]}{d t}=-\frac{\beta K}{C} i_{C}-\frac{v_{\mathrm{i}}-\bar{u} v_{\mathrm{o}}}{L} \\
\dot{x_{2}}=\frac{d\left[V_{\mathrm{ref}}-\beta v_{\mathrm{o}}\right]}{d t}=-\frac{\beta}{C} i_{C} \\
\dot{x_{3}}=x_{1}+x_{2} \\
\dot{x_{4}}=\int\left[x_{1}+x_{2}\right] d t
\end{array}\right.
$$

The equivalent control signal of the proposed system is obtained by solving $\frac{d S}{d t}=\alpha_{1} \dot{x_{1}}+\alpha_{2} \dot{x_{2}}+\alpha_{3} \dot{x_{3}}=0$ [10], which gives

$$
\begin{array}{r}
u_{\mathrm{eq}}=1-\frac{\beta L}{C v_{\mathrm{o}}}\left(K+\frac{\alpha_{2}}{\alpha_{1}}\right) i_{C}-\frac{v_{\mathrm{i}}}{v_{\mathrm{o}}}+\frac{\alpha_{3} L}{\alpha_{1} v_{\mathrm{o}}}\left(V_{\mathrm{ref}}-\beta v_{\mathrm{o}}\right) \\
+\frac{\alpha_{3} L}{\alpha_{1} v_{\mathrm{o}}}\left[K\left(V_{\mathrm{ref}}-\beta v_{\mathrm{o}}\right)-i_{L}\right]+\frac{\alpha_{4} L}{\alpha_{1} v_{\mathrm{o}}} \int\left(V_{\mathrm{ref}}-\beta v_{\mathrm{o}}\right) d t \\
+\frac{\alpha_{4} L}{\alpha_{1} v_{\mathrm{o}}} \int\left[K\left(V_{\mathrm{ref}}-\beta v_{\mathrm{o}}\right)-i_{L}\right] d t
\end{array}
$$

where $u_{\text {eq }}$ is continuous and bounded between 0 and 1 .

\section{B. Controller Architecture in PWM Form}

For implementation of indirect SM controller in PWM form, a set of equation comprising a control signal $v_{\mathrm{c}}$ and a ramp signal $\hat{v}_{\text {ramp }}$ must be derived using the indirect SM control technique [5]. In PWM form, the proposed DISM current controller for the boost converter inherits the expression

$$
\left\{\begin{aligned}
v_{\mathrm{c}}= & K_{1}\left(V_{\text {ref }}-\beta v_{\mathrm{o}}\right)+K_{2} \int\left(V_{\text {ref }}-\beta v_{\mathrm{o}}\right) d t \\
& +K_{1}\left[K\left(V_{\text {ref }}-\beta v_{\mathrm{o}}\right)-i_{L}\right] \\
& +K_{2} \int\left[K\left(V_{\text {ref }}-\beta v_{\mathrm{o}}\right)-i_{L}\right] d t \\
& -K_{3} i_{C}+G_{s}\left(v_{\mathrm{o}}-v_{\mathrm{i}}\right) \\
\hat{v}_{\text {ramp }} & =G_{s} v_{\mathrm{o}}
\end{aligned}\right.
$$

where $K_{1}=G_{s} \frac{\alpha_{3} L}{\alpha_{1}} ; K_{2}=G_{s} \frac{\alpha_{4} L}{\alpha_{1}} ;$ and $K_{3}=$ $G_{s} \frac{\beta L}{C}\left(K+\frac{\alpha_{2}}{\alpha_{1}}\right)$ are the fixed gain parameters in the proposed controller, and $0<G_{s}<1$ voltage level scaling factor. Assuming $\beta=G_{s}$, the analog implementation of the derived PWM-based DISM current controller for the boost converters is illustrated in Fig. 1.

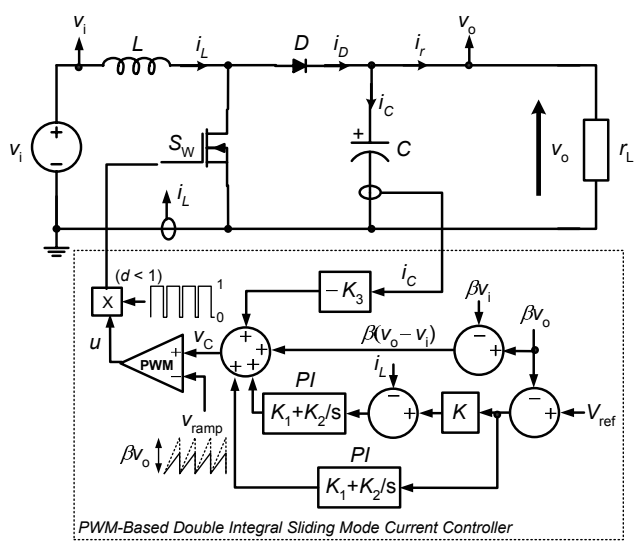

Fig. 1. Proposed PWM based DISM current controller for boost converters.

\section{Existence Condition}

The ranges of employable gain parameters $K_{n=1,2,3}$ for the controller design can be found by deriving the existence condition. For this purpose, the local reachability condition $\lim _{S \rightarrow 0} S \cdot \dot{S}<0$ must be satisfied. For the DISM current 
controlled boost converter, the existence condition for steadystate operation can be derived as

$$
\left\{\begin{array}{c}
-K_{3} i_{C(\min )}+K_{1}\left(x_{1(\max )}+x_{2(\max )}\right) \\
+K_{2} x_{3(\max )}<\beta v_{\mathrm{i}(\min )} \\
K_{3} i_{C(\max )}-K_{1}\left(x_{1(\min )}+x_{2(\min )}\right) \\
-K_{2} x_{3(\min )}<\beta\left(v_{\mathrm{o}(\mathrm{ss})}-v_{\mathrm{i}(\max )}\right)
\end{array}\right.
$$

where $v_{\mathrm{i}(\max )}$ and $v_{\mathrm{i}(\min )}$ denote the maximum and minimum input voltages respectively; $v_{\mathrm{o}(\mathrm{SS})}$ denotes the expected steadystate output, i.e., approximately the desired reference voltage $V_{\text {ref }}$; and $i_{C(\max )}$, and $i_{C(\min )}$ are respectively the maximum and minimum capacitor currents at full-load condition; $x_{1(\max )}$ and $x_{1(\min )}$ are respectively the maximum and minimum steady-state current errors; $x_{2(\max )}$ and $x_{2(\min )}$ are respectively the maximum and minimum steady-state voltage errors, which in this case are basically the inverse functions of the output voltage ripples; and $x_{3(\max )}$ and $x_{3(\min )}$ are respectively the maximum and minimum integrals of the combination of steady-state voltage and current errors. Fig. 2 illustrates the physical representations of these parameters. The design of control parameters must satisfy the inequalities in (13).

\section{Stability Condition}

The stability of the converter under the DISM controller can be achieved by making the eigenvalues of the Jacobian matrix of the system to have negative real parts. For the DISM current controlled boost converter, the motion equation is nonlinear. The stability condition is obtained by firstly deriving the ideal sliding dynamics of the system, and then doing an stability analysis on its equilibrium point [15].

1) Ideal Sliding Dynamics: The replacement of $\bar{u}$ by $\bar{u}_{\mathrm{eq}}$ (so-called equivalent control method) into the original boost converter's description under CCM operation converts the discontinuous system into an ideal SM continuous system:

$$
\left\{\begin{array}{l}
\frac{d i_{L}}{d t}=\frac{v_{\mathrm{i}}}{L}-\frac{v_{\mathrm{o}}}{L} \bar{u}_{\mathrm{eq}} \\
\frac{d v_{\mathrm{o}}}{d t}=\frac{i_{L}}{C} \bar{u}_{\mathrm{eq}}-\frac{v_{\mathrm{o}}}{r_{\mathrm{L}} C}
\end{array} .\right.
$$

Then, the substitution of the equivalent control signal into (14) gives (15), which represents the ideal sliding dynamics of the SM current controlled boost converter.

2) Equilibrium Point: Assume that there exists a stable equilibrium point on the sliding surface on which the ideal sliding dynamics eventually settled. At this point of equilibrium (steady state), there will not be any change in the system's dynamics if there is no input or loading disturbance, i.e., $\frac{d i_{L}}{d t}=\frac{d v_{\mathrm{o}}}{d t}=0$. Then, the state equations in (15) can be equated to give

$$
I_{L}=\frac{V_{\mathrm{o}}^{2}}{V_{\mathrm{i}} R_{\mathrm{L}}}
$$

where $I_{L}, V_{\mathrm{O}}, V_{\mathrm{i}}$, and $R_{\mathrm{L}}$ represents the inductor current, output voltage, input voltage, and load resistance at steadystate equilibrium, respectively.
3) Linearization of Ideal Sliding Dynamics: Next, the linearization of the ideal sliding dynamics around the equilibrium point transforms equation (15) into

$$
\left\{\begin{array}{l}
\frac{d \tilde{i}_{L}}{d t}=a_{11} \tilde{i}_{L}+a_{12} \int \tilde{i}_{L} d t+a_{13} \tilde{v}_{\mathrm{o}}+a_{14} \int \tilde{v}_{\mathrm{o}} d t \\
\frac{d \tilde{v}_{\mathrm{o}}}{d t}=a_{21} \tilde{i}_{L}+a_{22} \int \tilde{i}_{L} d t+a_{23} \tilde{v}_{\mathrm{o}}+a_{24} \int \tilde{v}_{\mathrm{o}} d t
\end{array}\right.
$$

where

$$
\left\{\begin{array}{l}
a_{11}=\frac{K_{2} V_{\mathrm{i}} R_{\mathrm{L}}}{K_{1} L V_{\mathrm{o}}-L V_{\mathrm{i}} R_{\mathrm{L}}} \\
a_{12}=\frac{K_{3} V_{\mathrm{i}} R_{\mathrm{L}}}{K_{1} L V_{\mathrm{o}}-L V_{\mathrm{i}} R_{\mathrm{L}}} \\
a_{13}=-\frac{K_{1} 2 V_{\mathrm{i}}-\frac{V_{\mathrm{i}}^{2} R_{\mathrm{L}}}{V_{\mathrm{o}}}-K_{2} K \beta \frac{V_{\mathrm{i}}}{R_{\mathrm{L}}}}{K_{1} L V_{\mathrm{o}}-L V_{\mathrm{i}} R_{\mathrm{L}}} \\
a_{14}=\frac{K_{3} K \beta \frac{V_{\mathrm{i}}}{R_{\mathrm{L}}}}{K_{1} L V_{\mathrm{o}}-L V_{\mathrm{i}} R_{\mathrm{L}}} \\
a_{21}=\frac{K_{1} V_{\mathrm{i}}-\frac{V_{\mathrm{i}}^{2} R_{\mathrm{L}}}{V_{\mathrm{o}}}-K_{2} V_{\mathrm{o}}}{K_{1} V_{\mathrm{o}} C-C V_{\mathrm{i}} R_{\mathrm{L}}} \\
a_{22}=\frac{K_{3} V_{\mathrm{o}}}{K_{1} V_{\mathrm{o}} C-C V_{\mathrm{i}} R_{\mathrm{L}}} \\
a_{23}=\frac{V_{\mathrm{i}}-K_{2} K \beta V_{\mathrm{o}}}{K_{1} V_{\mathrm{o}} C-C V_{\mathrm{i}} R_{\mathrm{L}}} \\
a_{24}=\frac{K_{3} K \beta V_{\mathrm{o}}}{K_{1} V_{\mathrm{o}} C-C V_{\mathrm{i}} R_{\mathrm{L}}}
\end{array}\right.
$$

The derivation is performed with the adoption of the following static equilibrium conditions, $V_{\mathrm{i}}=v_{\mathrm{i}}, R_{\mathrm{L}}=r_{\mathrm{L}}, V_{\text {ref }}-\beta V_{\mathrm{o}}=$ 0 , and $I_{\text {ref }}=I_{L}=K\left(V_{\text {ref }}-\beta V_{\mathrm{o}}\right)$, and the assumptions $K \gg 1, I_{L} \gg \tilde{i}_{L}$ and $V_{\mathrm{o}} \gg \tilde{v}_{\mathrm{o}}$. Therefore, the characteristic equation of the linearized system is given by

$$
\begin{aligned}
& \left|\begin{array}{cccc}
s-a_{11} & -a_{12} & -a_{13} & -a_{14} \\
-a_{21} & s-a_{22} & -a_{23} & -a_{24} \\
-1 & 0 & s & 0 \\
0 & 0 & -1 & s
\end{array}\right| \\
= & s^{4}+p_{1} s^{3}+p_{2} s^{2}+p_{3} s+p_{4}=0
\end{aligned}
$$

where

$$
\left\{\begin{array}{l}
p_{1}=-a_{11}-a_{21} \\
p_{2}=a_{11} a_{21}-a_{12} a_{21}-a_{13} \\
p_{3}=a_{13} a_{21}-a_{12} a_{23}-a_{14} \\
p_{4}=a_{14} a_{21}-a_{12} a_{24}
\end{array} .\right.
$$

The application of the Routh criterium to the characteristic equation (19) shows that the system will be stable if the following conditions are satisfied

$$
\left\{\begin{array}{l}
p_{1}>0 \\
p_{2}>p_{1} p_{4} \\
p_{3}>\frac{p_{1}^{2} p_{4}}{p_{1} p_{2}-p_{3}} \\
p_{4}>0
\end{array}\right.
$$

Hence, by numerically solving equation (18) and substituting its solutions into (21), the stability of the system can be determined. This, along with the existence condition (13), form the basis for the selection and design of the control gains of the proposed DISM controllers in terms of the converter's specification. The compliance of the stability condition assures that there exists an equilibrium point on the sliding surface, and the compliance of the existence condition assures that the state trajectory tracks the surface to this point of equilibrium. 


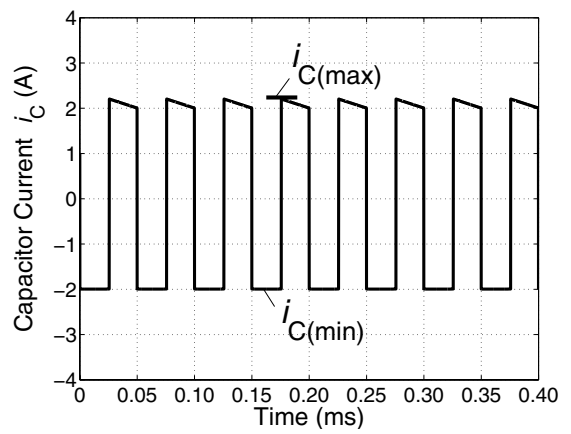

(a) Capacitor current $i_{\mathrm{C}}$

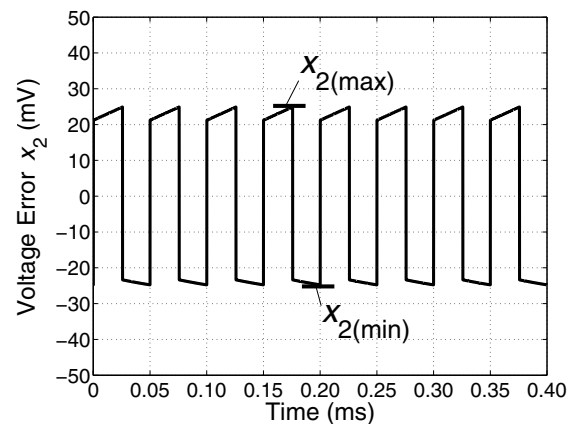

(c) Voltage error $x_{2}$

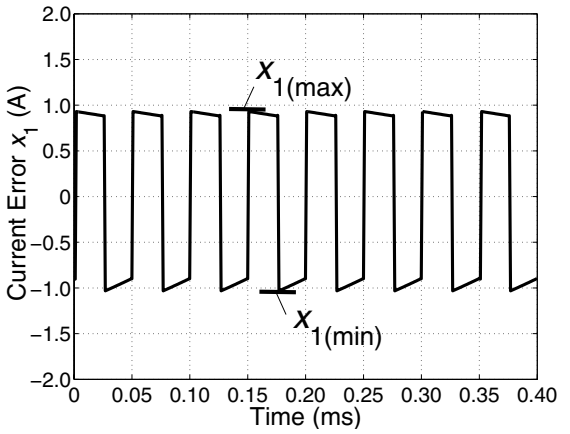

(b) Current error $x_{1}$

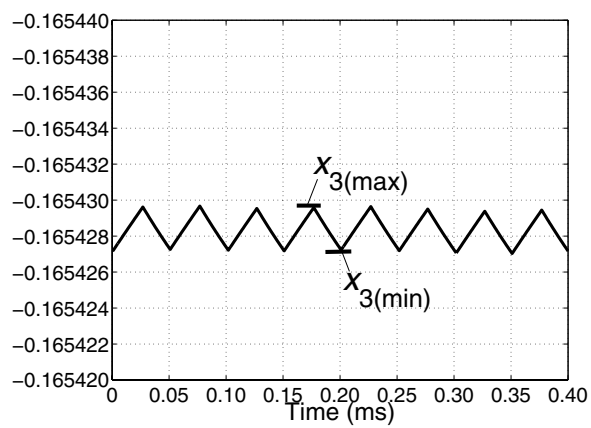

(d) Integral of sum of voltage and current errors $x_{3}$

Fig. 2. Steady-state waveforms of the various state variables of the boost converter at minimum input voltage and full-load condition under ideal open-loop control with negligible output voltage steady-state error.

$$
\left\{\begin{aligned}
\frac{d i_{L}}{d t} & =\frac{v_{\mathrm{i}}}{L}-\frac{v_{\mathrm{o}}}{L} \frac{K_{1} \frac{v_{\mathrm{o}}}{r_{\mathrm{L}}}-v_{\mathrm{i}}+K_{2}\left[V_{\mathrm{ref}}-\beta v_{\mathrm{o}}\right]+K_{2}\left[i_{\mathrm{ref}}-i_{L}\right]+K_{3} \int\left[V_{\mathrm{ref}}-\beta v_{\mathrm{o}}\right] d t+K_{3} \int\left[i_{\mathrm{ref}}-i_{L}\right] d t}{K_{1} i_{L}-v_{\mathrm{o}}} \\
\frac{d v_{\mathrm{o}}}{d t} & =\frac{i_{L}}{C} \frac{K_{1} \frac{v_{\mathrm{o}}}{r_{\mathrm{L}}}-v_{\mathrm{i}}+K_{2}\left[V_{\mathrm{ref}}-\beta v_{\mathrm{o}}\right]+K_{2}\left(i_{\mathrm{ref}}-i_{L}\right)+K_{3} \int\left[V_{\mathrm{ref}}-\beta v_{\mathrm{o}}\right] d t+K_{3} \int\left[i_{\mathrm{ref}}-i_{L}\right] d t}{K_{1} i_{L}-v_{\mathrm{o}}}-\frac{v_{\mathrm{o}}}{r_{\mathrm{L}} C}
\end{aligned}\right.
$$

TABLE I

SPECIFICATION OF Boost CONVERTER

\begin{tabular}{|lcc|}
\hline Description & Parameter & Nominal Value \\
\hline Input voltage & $v_{\mathrm{i}}$ & $24 \mathrm{~V}$ \\
Capacitance & $C$ & $220 \mu \mathrm{F}$ \\
Capacitor ESR & $r_{C}$ & $25 \mathrm{~m} \Omega$ \\
Inductance & $L$ & $300 \mu \mathrm{H}$ \\
Inductor resistance & $r_{L}$ & $0.14 \Omega$ \\
Switching frequency & $f_{\mathrm{S}}$ & $200 \mathrm{kHz}$ \\
Minimum output current & $i_{r(\min )}$ & $0.5 \mathrm{~A}$ \\
Maximum output current & $i_{r(\max )}$ & $2 \mathrm{~A}$ \\
Desired output voltage & $V_{\text {od }}$ & $48 \mathrm{~V}$ \\
\hline
\end{tabular}

\section{Results And Discussions}

The performance of the proposed PWM-based DISM current controlled boost converter shown in Fig. 1 is experimentally verified and compared to the PWM-based ISM current controlled boost converter [11]. The specification of the converter is given in Table I. Both the PWM-based ISM and DISM controller is optimally tuned to give the fastest critically-damped response [11]. The reference voltage is fixed at $V_{\text {ref }}=8 \mathrm{~V}$, which makes $\beta=G_{s}=0.167$. The control signal equation of the ISM controller is $v_{\mathrm{c}}=-i_{C}+$ $4.167\left(V_{\text {ref }}-0.167 v_{\mathrm{o}}\right)-0.77 i_{L}+0.167\left(v_{\mathrm{o}}-v_{\mathrm{i}}\right)$ and the control signal ${ }^{1}$ equation of the DISM controller is $v_{\mathrm{c}}=-i_{C}+$ $1.58\left(V_{\text {ref }}-0.167 v_{\mathrm{o}}\right)+1220 \int\left(V_{\text {ref }}-0.167 v_{\mathrm{o}}\right) d t-0.77 i_{L}+$ $0.167\left(v_{\mathrm{o}}-v_{\mathrm{i}}\right)$. The difference between the two equations is the additional integral term of $1220 \int\left(V_{\text {ref }}-0.167 v_{\mathrm{o}}\right) d t$ and the choice of a lower gain value (i.e., 1.58) of $V_{\text {ref }}-0.167 v_{\mathrm{o}}$ in the DISM controller. The reduction in the proportional gain is to tackle the instability due to the introduction of the integral pole term.

Figs. 3(a) and 3(b) show the plots of the steady-state output voltage against the switching frequency of the boost converter under the PWM-based ISM and DISM controllers for respectively the minimum and maximum output currents. It is shown that the addition of the double-integral term of the state variables into the PWM ISM controller reduces the steady-state regulation error (between minimum and maximum output currents) from $<1 \%$ to $<0.05 \%$ for all values of switching frequency. Hence, the strength of the proposed solution in alleviating the steady-state error of PWM ISM controller is illustrated.

Figs. 4(a) and 4(b) show the output voltage waveforms of the boost converter operating at $200 \mathrm{kHz}$ and step load changes alternating between $0.5 \mathrm{~A}$ and $2 \mathrm{~A}$. As seen from the figures,

\footnotetext{
${ }^{1}$ The implementation ignores the inductor current error, which is negligible because of the high current-error gain value of $K$.
} 


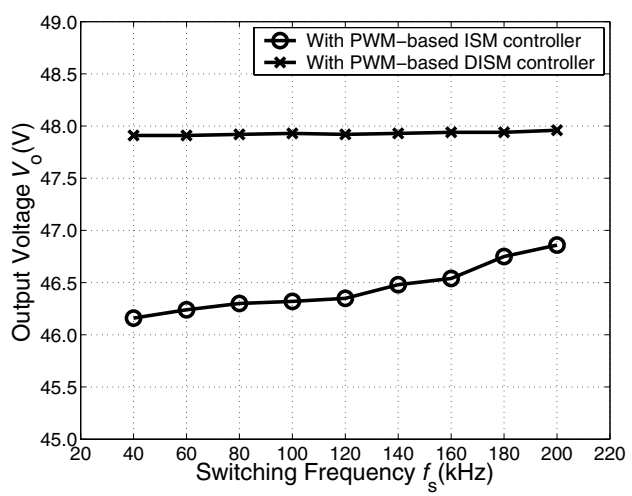

(a) At minimum output current $i_{r}=0.5 \mathrm{~A}$

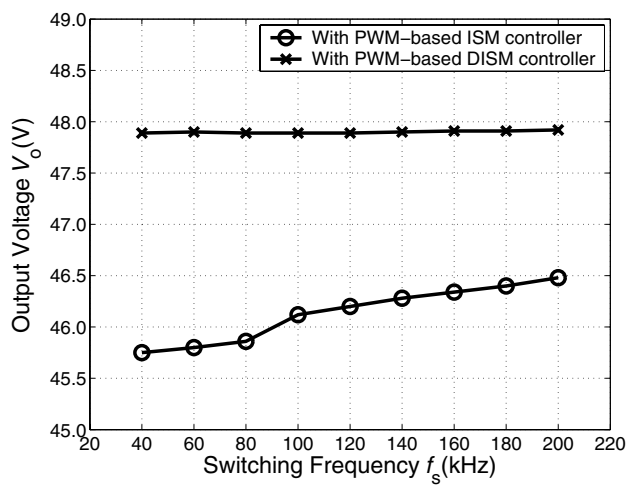

(b) At maximum output current $i_{r}=2.0 \mathrm{~A}$

Fig. 3. Plot of steady-state output voltage $v_{\mathrm{o}}$ against switching frequency $f_{\mathrm{s}}$ of the boost converter operating under the PWM-based ISM and DISM controllers at (a) minimum and (b) maximum output currents.

both controllers displayed excellent large-signal property (a major feature of the SM control) of having a consistent response for both step up and down load changes. However, with the PWM-based ISM controller, the converter contains a significant level of steady-state error of around $400 \mathrm{mV}$ (see Fig. 4(a)). Such error is not present with the PWMbased DISM controller (see Fig. 4(b)). Yet, it can be seen that with the PWM-based ISM controller, a faster dynamical response can be achieved. This is due to the higher value of the voltage-error control gain in the PWM-based ISM controller (gain value of 4.167) than the PWM-based DISM controller (gain value of 1.58). Hence, it is important to remarked that even though the steady-state error of the PWM-based ISM controller can be alleviated to achieve near perfect regulation by converting it into the DISM controller, it may come at the expense of a lower allowable proportional gain value, which gives a slower dynamical response.

\section{CONCLUSION}

In this paper, the failure of the integral control scheme in the indirect form of ISM controllers in alleviating the steadystate regulation error of power converters has been examined. It has been found that the problem of the method of steadystate error correction in these controllers lies in the use of an indirect method of computing the integral of the steady-state error. Notably, the magnitude of the regulation error increases

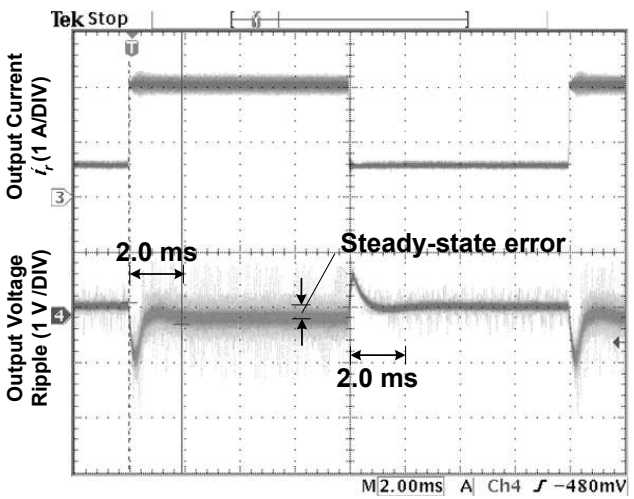

(a) ISM controller

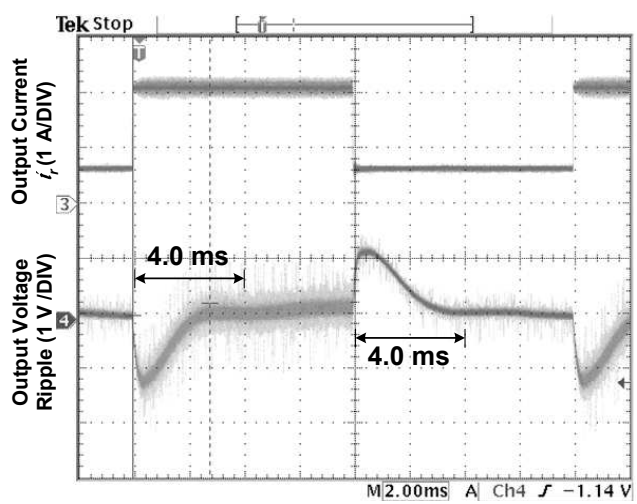

(b) DISM controller

Fig. 4. Output voltage waveforms of a $24 \mathrm{~V}$ input, $48 \mathrm{~V}$ output boost converter operating at step load changes alternating between $0.5 \mathrm{~A}$ and $2 \mathrm{~A}$ under (a) the PWM-based ISM controller; and (b) the PWM-based DISM controller.

as the switching frequency reduces, and it becomes prominent at the low frequency range. In view of this, it is proposed that a double-integral term of the controlled variables can be added for constructing the sliding surface of indirect SM controllers. The inclusion of this additional term is to correct the error of the indirect integral computation. By doing so, the regulation error of the converter is indirectly alleviated. Experimental results show that the proposed DISM controller is capable of achieving a near perfect regulation even at a low switching frequency.

\section{REFERENCES}

[1] S.C. Tan, Y.M. Lai, C.K. Tse, and M.K.H. Cheung, "Adaptive feedforward and feedback control schemes for sliding mode controlled power converters", IEEE Transactions on Power Electronics, vol. 21 no. 1, pp. 182-192, Jan. 2006.

[2] B.J. Cardoso, A.F. Moreira, B.R. Menezes, and P.C. Cortizo, "Analysis of switching frequency reduction methods applied to sliding mode controlled DC-DC converters", in Proceedings, IEEE Applied Power Electronics Conference and Exposition (APEC), pp. 403-410, February 1992.

[3] P. Mattavelli, L. Rossetto, G. Spiazzi, and P. Tenti, "General-purpose sliding-mode controller for dc/dc converter applications", in IEEE Power Electronics Specialists Conference Record (PESC), pp. 609-615, June 1993.

[4] V.M. Nguyen and C.Q. Lee, "Indirect implementations of sliding-mode control law in buck-type converters", in Proceedings, IEEE Applied Power Electronics Conference and Exposition (APEC), vol. 1, pp. 111115, March 1996. 
[5] S.C. Tan, Y.M. Lai, C.K. Tse, and M.K.H. Cheung, "A fixed-frequency pulse-width-modulation based quasi-sliding mode controller for buck converters", IEEE Transactions on Power Electronics, vol. 20, no. 6, pp. 1379-1392, Nov. 2005.

[6] S.C. Tan, Y.M. Lai, and C.K. Tse, "A unified approach to the design of PWM based sliding mode voltage controller for basic DC-DC converters in continuous conduction mode", IEEE Transactions on Circuits and Systems I: Fundamental Theory and Applications, vol. 53, no. 8, pp. 1816-1827 Aug. 2006.

[7] M. Castilla, L.C. de Vicuna, M. Lopez, O. Lopez, and J. Matas, "On the design of sliding mode control schemes for quantum resonant converters", IEEE Transactions on Power Electronics, vol. 15 no. 15, pp. 960-973, Nov. 2000.

[8] S.K. Mazumder, A.H. Nayfeh, A. Borojevic, P. Gaya, and O. López, "Robust control of parallel DC-DC buck converters by combining integral-variable-structure and multiple-sliding-surface control schemes", IEEE Transactions on Power Electronics, vol. 17, no. 3, pp. 428-437, May 2002.

[9] H. Sira-Ramirez, "On the generalized PI sliding mode control of DCto-DC power converters: a tutorial", International Journal of Control, vol. 76 no. 9/10, pp. 1018-1033, 2003.

[10] V. Utkin, J. Guldner, and J.X. Shi, Sliding Mode Control in Electromechanical Systems. London, U.K.: Taylor and Francis, 1999.

[11] S.C. Tan, Y.M. Lai, C.K. Tse, and C.K. Wu, "A pulsewidth modulation based integral sliding mode current controller for boost converters", in IEEE Power Electronics Specialists Conference Record (PESC 2006), pp. 1612-1618, June 2006, Jeju, Korea.

[12] J. Mahdavi, A. Emadi, and H.A. Toliyat, "Application of state space averaging method to sliding mode control of PWM DC/DC converters," in Proceedings, IEEE Conference on Industry Applications (IAS), vol. 2, pp. 820-827, Oct. 1997.

[13] J. Mahdavi, M.R. Nasiri, and A. Agah, "Application of neural networks and state space averaging to a DC/DC PWM converter in sliding mode operation," in Proceedings, IEEE Conference on Industrial Electronics, Control and Instrumentations (IECON), vol. 1, pp. 172-177, 2000.

[14] K. Ogata, Modern Control Engineering. Upper Saddle River, N.J.: Prentice-Hall, Inc., 1997.

[15] L. Martinez-Salamero, J. Calvente, R. Giral, A. Poveda, and E. Fossas, "Analysis of a bidirectional coupled-inductor Ćuk converter operating in sliding mode," IEEE Transactions on Circuits and Systems Part I: Fundamental Theory and Applications, vol. 45, no. 4, pp. 355-363, April 1998. 